\title{
FINITE MORPHISMS ONTO FANO MANIFOLDS OF PICARD NUMBER 1 WHICH HAVE RATIONAL CURVES WITH TRIVIAL NORMAL BUNDLES
}

\author{
JUN-MUK HWANG AND NGAIMING MOK
}

\begin{abstract}
Let $X$ be a Fano manifold of Picard number 1 admitting a rational curve with trivial normal bundle and $f: X^{\prime} \rightarrow X$ be a generically finite surjective holomorphic map from a projective manifold $X^{\prime}$ onto $X$. When the domain manifold $X^{\prime}$ is fixed and the target manifold $X$ is a priori allowed to deform we prove that the holomorphic map $f: X^{\prime} \rightarrow X$ is locally rigid up to biholomorphisms of target manifolds. This result complements, with a completely different method of proof, an earlier local rigidity theorem of ours (see J. Math. Pures Appl. 80 (2001), 563575 ) for the analogous situation where the target manifold $X$ is a Fano manifold of Picard number 1 on which there is no rational curve with trivial normal bundle. In another direction, given a Fano manifold $X^{\prime}$ of Picard number 1, we prove a finiteness result for generically finite surjective holomorphic maps of $X^{\prime}$ onto Fano manifolds (necessarily of Picard number 1) admitting rational curves with trivial normal bundles. As a consequence, any 3-dimensional Fano manifold of Picard number 1 can only dominate a finite number of isomorphism classes of projective manifolds.
\end{abstract}

There are various results concerning finiteness or rigidity of dominant morphisms or rational maps from a fixed projective variety onto varieties of nonpositive curvature. For example, Kobayashi-Ochiai showed ([KO]) that for any algebraic variety $Y$ and any algebraic variety $Z$ of general type, the number of dominant rational maps from $Y$ to $Z$ is finite. Another example is Maehara's result ([Ma] $)$ that for a given projective algebraic manifold $Y$, the number of minimal projective manifolds of general type, which can be the image of a dominant rational map from $Y$, is finite. Eckart Viehweg had informed us that the works on the semi-positivity of direct images of tensor powers of dualizing sheaves, due to Kawamata, Viehweg and others, imply that for any projective algebraic variety $Y$, there are only countably many

Received December 18, 2000. Supported by Grant No. 98-0701-01-5-L from the KOSEF. Supported by a grant of the Hong Kong Research Grants Council. 
minimal (not necessarily of general type) projective manifolds which can be the image of $Y$ under a morphism.

On the other hand, there are very few results of this type when the targets have positive curvature, i.e. when the targets are Fano manifolds. In HM1, the authors showed that a projective manifold, which can be the image of a rational homogeneous space $G / P$ of Picard number 1 under a holomorphic map, must be either $G / P$ itself or the projective space. As a natural generalization of this result, one may ask which projective manifolds (which are automatically Fano) can be the image of a holomorphic map from a fixed Fano manifold $Y$ of Picard number 1. However, for a general $Y$, the possible images can be more than just itself or the projective space, as one can easily see in the case of some Fano hypersurfaces in the projective space. So it seems that a more modest question about the finiteness of such images is already worth studying:

Question. Given a Fano manifold $Y$ of Picard number 1, are there only a finite number of isomorphism classes of projective manifolds which can be the image of $Y$ under a holomorphic map?

One of the results of this paper is an affirmative answer to the Question in dimension 3. Our main results hold in any dimension, but we need to impose an additional condition on the image manifolds. Let us explain this condition.

Let $X$ be a Fano manifold of Picard number 1. It is easy to see from the deformation theory of rational curves (e.g. $[\mathrm{Kl}]$ ) that the following conditions on $X$ are equivalent.

(1) There exists a rational curve $\mu: \mathbf{P}_{1} \rightarrow X$ so that $\mu^{*} T(X) \cong \mathcal{O}(2) \oplus$ $\mathcal{O}^{n-1}$.

(2) For a generic point $x \in X$, there are only finitely many rational curves through $x$ which have minimal degree with respect to $K_{X}^{-1}$.

(3) For a generic point $x \in X$, there exists a rational curve which has degree 2 with respect to $K_{X}^{-1}$.

A rational curve satisfying the first condition will be called a rational curve with trivial normal bundle. The rational curve in the second and the third conditions is a rational curve with trivial normal bundle.

There are many examples of Fano manifolds which satisfy the above equivalent conditions. When $n=3$, excepting the projective space $\mathbf{P}_{3}$ and the 3-dimensional hyperquadric, all Fano 3-folds of Picard number 1 satisfy the condition (【s $)$. Also, for any $m \geq 4$, a smooth hypersurface of degree $m-1$ or $m$ in $\mathbf{P}_{m}$ satisfies it. When the degree is $m-1$, there are finitely many lines through a generic point. When the degree is $m$, there exist no lines through a generic point, but finitely many conics through a generic point. 
In this paper, we will study finite morphisms over Fano manifolds of Picard number 1 which have rational curves with trivial normal bundles. We have two main results. The first one is the deformation rigidity of such morphisms in the following sense:

Theorem 1. Let $\chi: \mathcal{X} \rightarrow \Delta:=\{t \in \mathbf{C},|t|<1\}$ be a regular family of Fano manifolds of Picard number 1 so that $X_{0}=\chi^{-1}(0)$ has rational curves with trivial normal bundles. For a given projective manifold $X^{\prime}$, suppose there exists a surjective morphism $f: \mathcal{X}^{\prime}=X^{\prime} \times \Delta \rightarrow \mathcal{X}$ respecting the projections to $\Delta$ so that $f_{t}: X^{\prime} \rightarrow X_{t}$ is a generically finite morphism for each $t \in \Delta$. Then there exists $\epsilon>0$ and a holomorphic family of biholomorphic morphisms $v_{t}: X_{o} \rightarrow X_{t},|t|<\epsilon$, satisfying $f_{t}=v_{t} \circ f_{o}, v_{0}=i d$.

In particular, the set $\operatorname{Hol}\left(X^{\prime}, X\right)$ of generically finite morphisms from a complete variety $X^{\prime}$ onto $X$ is countable up to automorphisms of $X$. An analogous result was obtained in [HM3] for a class of Fano manifolds of Picard number 1 which do not have rational curves with trivial normal bundles. The methods employed in HM3 cannot be applied to the current case at all and have little to do with the techniques used in this paper.

Our second result is the boundedness of the degrees of the finite morphisms when the domain $X^{\prime}$ is also a Fano manifold of Picard number 1.

Theorem 2. Let $X$ and $X^{\prime}$ be $n$-dimensional Fano manifolds of Picard number 1. Assume that $X$ has rational curves with trivial normal bundles. Then there exists a positive number $N$ determined by $X^{\prime}$ such that for any finite morphism $f: X^{\prime} \rightarrow X$, the degree of $f$ is bounded by $N$.

As a consequence of Theorems 1 and 2, we obtain the following partial answer to the Question.

Corollary 4. Let $X^{\prime}$ be a Fano manifold of Picard number 1. Amid Fano manifolds of Picard number 1 which have rational curves with trivial normal bundles, only finitely many can be the image of a holomorphic map from $X^{\prime}$. Furthermore, for each such $X$ there are at most a finite number of non-trivial holomorphic maps $f: X^{\prime} \rightarrow X$ up to automorphisms of $X$.

As mentioned above, among Fano threefolds only the hyperquadric and the projective space do not have rational curves with trivial normal bundles. Thus, Corollary 4 gives an affirmative answer to the Question in dimension 3.

It should be mentioned that Theorem 2 was proved in dimension 3 by $\mathrm{Am}$ and $[\mathrm{ARV}]$. In fact, they proved the same result for any (not necessarily Fano) projective threefold $X^{\prime}$ of Picard number 1. In dimension 3, many additional results on morphisms to Fano threefolds were obtained in Am, ARV], [IS] and $[\mathrm{Sc}$ ], many of which cannot be covered by our results. On the other hand, their methods rely heavily on the classification of Fano threefolds while ours is basically independent of the classification theory. Moreover, their methods do 
not seem applicable to the question of local rigidity (Theorem 1 ). For example, our Corollary 2, that finite morphisms from Mukai-Umemura threefolds onto Fano threefolds different from $\mathbf{P}_{3}$ must be isomorphisms, is out of reach of their methods.

Our approach is based on the philosophy that many problems on Fano manifolds can be solved by looking at tangent directions to minimal rational curves (see HM1, HM2 for other examples of this philosophy). The tangent directions to rational curves with trivial normal bundles form a finite branched covering of the Fano manifold. The main idea of the paper is to study the geometry of the branch locus of this covering and examine how it changes under a finite morphism. The crucial point in applying this study to problems on generically finite morphisms is that the inverse image of rational curves with trivial normal bundles under a generically finite morphism also have trivial normal bundles (Proposition 6). In general, these inverse images are not rational curves. So it is necessary to study curves with trivial normal bundles of arbitrary genus. Compared with rational curves, the deformation theory of curves of high genus could be quite tricky. However, in the situation we are studying, no obstructions appear and the deformation theory is quite parallel to that of rational curves with trivial normal bundles. The first two sections present a general theory of curves with trivial normal bundles covering a projective manifold. The next two sections study its behavior under generically finite morphisms. The proof of Theorem 1, which will be given in Section 5, uses only Section 1 and Section 3 and is independent of Section 2 and Section 4. The proof of Theorem 2 will be given in Section 6 .

\section{Convention and terminology}

1. All varieties and morphisms are defined over complex numbers. Open sets of a variety mean Euclidean open sets, not Zariski open sets. A variety need not be irreducible, but has finitely many components. A generic point of a variety of pure dimension means a generic point of any of its components. So when we say that a certain statement holds for a generic point of a variety of pure dimension, it means that it holds for a generic point of each component of the variety.

2. A projective manifold means an irreducible smooth projective variety. A Fano manifold means a projective manifold with ample anti-canonical bundle.

3. For a proper morphism between two varieties $\varphi: Y \rightarrow X$, the inverse image $\varphi^{-1}(Z)$ of a subvariety $Z \subset X$ is taken in the set-theoretical sense and understood as a reduced subvariety of $Y$. 
4. Suppose a generically finite morphism $\varphi: Y \rightarrow X$, from a complete variety $Y$ to a projective manifold $X$, is given. Consider the set

$$
\{y \in Y, Y \text { is singular at } y \text { or } \varphi \text { is ramified at } y\} \text {. }
$$

The ramification divisor of $\varphi$ is the union of codimension- 1 components of the above set which are not contracted to lower dimensional subvarieties of $X$ by $\varphi$. The branch divisor of $\varphi$ is the image of the ramification divisor. Let $B \subset X$ be the branch divisor. Then $\varphi$ is unramified over $X-B-Z$ for some subvariety $Z \subset X$ of codimension $>1$. For a point $y \in Y$ where $\varphi$ is locally finite, the degree of the germ of $\varphi$ at $y$ will be called the local sheeting number of $\varphi$ at $y$. Given a point $x \in X$, an isolating neighborhood of $x$ with respect to $\varphi$ means a connected open subset $U \subset X$ containing $x$ such that for each irreducible component $G$ of $\varphi^{-1}(U), G \cap \pi^{-1}(x)$ is a single point. There exists a subvariety of codimension $>1$ in $X$ so that any point outside this subvariety has an isolating neighborhood.

\section{Webs and their discriminantal divisors}

Let $X$ be a projective manifold of dimension $n$ and $C \subset X$ be an irreducible reduced curve. We say that $C$ has a trivial normal bundle if under the normalization $\mu: \tilde{C} \rightarrow C$, we get the following exact sequence

$$
0 \longrightarrow \Theta_{\tilde{C}} \longrightarrow \mu^{*} \Theta_{X} \longrightarrow \mathcal{O}_{\tilde{C}}^{n-1} \longrightarrow 0
$$

where $\Theta$ denotes the tangent sheaf. In this case, $\mu: \tilde{C} \rightarrow X$ is an immersion. Suppose that $C$ has a trivial normal bundle and deformations of $C$ with constant geometric genus cover an open subset of $X$. The germ of the space of deformations of $C$ with constant geometric genus must have dimension $\geq n-1$. The Zariski tangent space to this space at the point corresponding to $C$ is $H^{0}\left(\tilde{C}, \mu^{*} \Theta_{X} / \Theta_{\tilde{C}}\right)$ (cf. [HM1], p. 212), which has dimension $n-1$ from the triviality of the normal bundle. Thus the germ is smooth. The closure $\mathcal{M}_{C}$ of this germ in the Hilbert scheme of $X$ will be called the irreducible web defined by $C$. The underlying variety of $\mathcal{M}_{C}$ is irreducible, projective, of dimension $n-1$ and smooth at the point $[C]$ corresponding to $C$. Conversely, an irreducible subscheme of dimension $n-1$ in the Hilbert scheme of curves on $X$ is called an irreducible web if its members cover $X$ and generic members have trivial normal bundles. A web is a finite collection of irreducible webs. There are only finitely many irreducible webs in an irreducible component of the Hilbert scheme of curves on $X$. Thus there are only countably many webs for a given $X$. 
For a web $\mathcal{M}$, let $\psi: F \rightarrow \mathcal{M}$ and $\phi: F \rightarrow X$ be the universal family morphisms. We will assume that $F$ and $\mathcal{M}$ are normal projective varieties by taking the normalization of the underlying reduced structures of all the schemes involved. Then $\psi$ need not be flat any more, but every fiber of $\psi$ is of pure dimension 1 and a generic fiber of $\psi$ is an irreducible smooth curve. Note that $F$ and $\mathcal{M}$ are varieties of pure dimension. Clearly, $\phi$ is generically finite. If $\phi$ is $d$-to- $1, d$ is called the degree of the web $\mathcal{M}$. In a neighborhood of a generic point of $X$, the images of fibers of $\psi$ define $d$ distinct local foliations by open pieces of curves.

Remark. In local differential geometry, a finite collection of foliations of equal rank is called a web. At a generic point of $X$ a web in our sense defines a web of rank-1 in the differential-geometric sense.

From the triviality of the normal bundle, $\phi$ is unramified in a neighborhood of a generic fiber of $\psi$. It follows that for a given subvariety $Z \subset X$ of codimension $>1$, a generic member of $\mathcal{M}$ is disjoint from $Z$.

We say that a connected curve $C$ in a smooth variety is transversal to a hypersurface $H$ if either $C \cap H$ is empty or it consists of finitely many smooth points of $H$ where each local irreducible germ of $C$ is smooth and is not tangent to $H$.

Proposition 1. Let $\mathcal{M}$ be a web on $X$. Let $H \subset X$ be an irreducible hypersurface and $y \in H$ be a generic point. Then for any $v \in \mathcal{M}$ with $y \in$ $\phi\left(\psi^{-1}(v)\right)$, one of the following holds.

(i) $\phi\left(\psi^{-1}(v)\right)$ has trivial normal bundle and is transversal to $H$.

(ii) $\phi\left(\psi^{-1}(v)\right)$ is locally irreducible at $y$ and its component containing $y$ is contained in $H$.

Proof. Since $\phi$ is unramified in a neighborhood of a generic fiber of $\psi$, the statement (i) holds for a generic $v \in \mathcal{M}$. Let

$$
\mathcal{N}:=\{v \in \mathcal{M} \text {, (i) does not hold for } v\} .
$$

Then $\operatorname{dim}(\mathcal{N}) \leq n-2$ and $\phi\left(\psi^{-1}(\mathcal{N})\right)$ is a proper subvariety of $X$. When $H \not \subset \phi\left(\psi^{-1}(\mathcal{N})\right)$, choose $y \in H-\phi\left(\psi^{-1}(\mathcal{N})\right)$. Then for any $v \in \mathcal{M}$ with $y \in$ $\phi\left(\psi^{-1}(v)\right)$, (i) is always satisfied. When $H \subset \phi\left(\psi^{-1}(\mathcal{N})\right)$, let $\phi\left(\psi^{-1}(\mathcal{N})\right)=$ $H \cup G$ where $G$ consists of components different from $H$ and choose $y \in H-G$. Then for any $v \in \mathcal{M}$ with $y \in \phi\left(\psi^{-1}(v)\right)$, either Case (1) $v \notin \mathcal{N}$ and (i) holds, or Case (2) $v \in \mathcal{N}$ and $\phi\left(\psi^{-1}(v)\right) \subset H \cup G$. In Case (2), an irreducible component of $\phi\left(\psi^{-1}(v)\right)$ containing $y$ is contained in $H$. Since $\operatorname{dim}(\mathcal{N}) \leq n-2$, such a curve is locally irreducible at a generic point of $H$.

Let $\Phi: F \rightarrow \mathbf{P} T(X)$ be the rational map defined by

$$
\Phi(a):=\mathbf{P} T_{\phi(a)}\left(\phi\left(\psi^{-1}(\psi(a))\right)\right)
$$


for a generic point $a \in F$. Namely, $\Phi(a)$ is the tangent direction of the curve corresponding to $\psi(a) \in \mathcal{M}$ at the point $\phi(a)$. $\Phi$ will be called the tangent map. Let $\mathcal{C} \subset \mathbf{P} T(X)$ be the strict image of $\Phi$. $\mathcal{C}$ will be called the variety of $\mathcal{M}$-tangents.

Proposition 2. Let $\pi: \mathcal{C} \rightarrow X$ be the restriction of the projection $\mathbf{P} T(X)$ $\rightarrow X$ to $\mathcal{C}$. Then $\pi$ is generically d-to-1, where $d$ is the degree of the web. Moreover, there exists a subvariety $E$ of codimension $\geq 2$ in $X$ so that $\Phi$ is well-defined on $F-\phi^{-1}(E)$ and can be regarded as the normalization of $\left.\mathcal{C}\right|_{X-E}$.

Proof. Suppose $\pi: \mathcal{C} \rightarrow X$ is generically $m$-to- 1 for $m \leq d$. At a generic point $x \in X$ where $\pi$ is unramified, $\mathcal{C} \subset \mathbf{P} T(X)$ defines $m$ distinct local foliations by open pieces of curves. This must agree with $d$ distinct foliations by open pieces of curves defined by the $\phi$-images of the $\psi$-fibers. Thus $m=d$. It follows that $\Phi: F \rightarrow \mathcal{C}$ is birational. Since $\Phi$ respects the morphisms $\phi: F \rightarrow X$ and $\pi: \mathcal{C} \rightarrow X$, the second statement is immediate.

The branch divisor of $\phi: F \rightarrow X$ will be called the discriminantal divisor of the web $\mathcal{M}$ and denoted by $D_{\mathcal{M}}$. The branch divisor of $\pi: \mathcal{C} \rightarrow X$ will be called the extended discriminantal divisor of $\mathcal{M}$ and denoted by $E_{\mathcal{M}}$. From Proposition 2, it is clear that $D_{\mathcal{M}} \subset E_{\mathcal{M}}$. When $y$ is a smooth point of $D_{\mathcal{M}}$, let $\mathbf{P} T_{y}\left(D_{\mathcal{M}}\right)$ be the projectivized tangent space. Let $\mathbf{P} T\left(D_{\mathcal{M}}\right) \subset \mathbf{P} T(X)$ be the subvariety defined as the closure of the union of $\mathbf{P} T_{y}\left(D_{\mathcal{M}}\right)$ as $y$ varies over the smooth points of $D_{\mathcal{M}}$. The variety $\mathbf{P} T\left(E_{\mathcal{M}}\right)$ is defined similarly.

Proposition 3. Let $\mathcal{R} \subset F$ be the ramification divisor of $\phi: F \rightarrow X$. Then $\Phi(\mathcal{R}) \subset \mathbf{P} T\left(D_{\mathcal{M}}\right)$.

Proof. Recall that $F$ and $\mathcal{M}$ are normal varieties. Since $\phi$ is unramified in a neighborhood of a generic fiber of $\psi$, a generic fiber of $\psi$ is disjoint from $\mathcal{R}$ and $\mathcal{R}$ consists of certain components of fibers of $\psi$ over a hypersurface in $\mathcal{M}$. These components of fibers of $\psi$ are sent to curves in $D_{\mathcal{M}}=\phi(\mathcal{R})$. Generic points of $\Phi(\mathcal{R})$ correspond to tangent vectors to these curves, hence $\Phi(\mathcal{R}) \subset \mathbf{P} T\left(D_{\mathcal{M}}\right)$ by Proposition 1.

\section{Discriminantal order}

Let $\mathcal{M}$ be a web on a projective manifold $X$. In this section, we will define integers $\delta_{L}$ for certain components $L$ of the extended discriminantal divisor $E_{\mathcal{M}} \subset X$.

Let $L$ be an irreducible component of $E_{\mathcal{M}}$ and let $y \in L$ be a generic point. Let $U$ be an isolating neighborhood of $y$ with respect to the generically finite 
morphism $\pi: \mathcal{C} \rightarrow X$ (cf. Convention and Terminology, 4). Let $\mathcal{G}$ be the union of components of $\left.\mathcal{C}\right|_{U}$ which intersect $\mathbf{P} T_{y}(L)$. When $\mathcal{G}$ is non-empty, it is generically $m$-to- 1 over $U$ for some positive integer $m$. This $m$ is certainly independent of the choice of the generic point $y$ and depends only on the choice of the component $L$ of $E_{\mathcal{M}}$. We will call it the tangential sheeting number of $\mathcal{M}$ along $L$ and denote it by $\tau_{L}$.

Proposition 4. If $M$ is a component of $D_{\mathcal{M}}$, then $\tau_{M}>1$.

Proof. Let $\mathcal{R}$ be a component of the ramification divisor of $\phi: F \rightarrow X$ over $M$. For a generic point $y$ of $M$, any germ of $\mathcal{R}$ over $y$ is smooth because $F$ is normal. Since $\phi$ is ramified at $y$, the local sheeting number $>1$. By Proposition 3, $\Phi(\mathcal{R})$ is contained in $\mathbf{P} T(M)$. Thus for a generic point $y \in M$, any germ of $\mathcal{C}$ at a generic point of $\Phi(\mathcal{R})$ gives a germ of $\mathcal{G}$ where the local sheeting number of $\pi$ is strictly bigger than 1 .

Suppose $L$ is a component of $E_{\mathcal{M}}$ with $\tau_{L}>1$. Let $\xi$ be the tautological line bundle on the projectivized tangent bundle $\mathbf{P} T(X)$. By shrinking $U$ if necessary, we may assume that $\xi$ is trivial on $\mathcal{G}$. Let $V$ be a non-vanishing section of $\xi$ over $\tilde{\mathcal{G}}$, the normalization of $\mathcal{G}$. In terms of a coordinate system $\left(z_{1}, \ldots, z_{n}\right)$ centered at $y \in L, V$ can be written as

$$
V=v_{1} \frac{\partial}{\partial z_{1}}+\cdots+v_{n} \frac{\partial}{\partial z_{n}}
$$

where $v_{i}$ 's are multi-valued holomorphic functions on $U$, or more precisely, holomorphic functions on $\tilde{\mathcal{G}}$. Set $m=\tau_{L}$. For a generic point $x \in U$, let $x^{1}, \ldots, x^{m}$ be the points of $\mathcal{G}$ over $x$. As $x$ approaches $y$, at least two of $x^{1}, \ldots, x^{m}$ get closer from the definition of $E_{\mathcal{M}}$. For an antisymmetric $n \times n$ complex matrix $Q=\left(q^{i j}\right) \in \mathbf{o}(n, \mathbf{C})$, consider the holomorphic function on $U$ defined by

$$
\Gamma_{Q}(x):=\prod_{1 \leq \alpha \neq \beta \leq m}\left(\sum_{i, j=1}^{n} q^{i j} v_{i}\left(x^{\alpha}\right) v_{j}\left(x^{\beta}\right)\right)
$$

for generic $x \in U$. Let $\gamma_{Q} \geq 1$ be the vanishing order of $\Gamma_{Q}$ along $L$ and set

$$
\delta_{y}:=\min _{Q \in \mathbf{o}(n, \mathbf{C})} \gamma_{Q} .
$$

Proposition 5. The positive integer $\delta_{y}$ is uniquely determined by the choice of $y \in L$, namely, it is independent of the choice of a local coordinate system at $y$ and the choice of the multi-valued vector field $V$.

Proof. First, we will show that once $V$ is chosen, then $\delta_{y}$ is independent of the choice of coordinates. Let $\Gamma_{Q}^{\sharp}, \gamma_{Q}^{\sharp}$ and $\delta_{y}^{\sharp}$ be as defined above using another coordinate system $\left(z_{1}^{\sharp}, \ldots, z_{n}^{\sharp}\right)$ given by $z_{i}^{\sharp}=\Psi_{i}\left(z_{1}, \ldots, z_{n}\right)$ for some 
holomorphic functions $\Psi_{i}, 1 \leq i \leq n$. Let

$$
V=v_{1}^{\sharp} \frac{\partial}{\partial z_{1}^{\sharp}}+\cdots+v_{n}^{\sharp} \frac{\partial}{\partial z_{n}^{\sharp}} .
$$

Then we have

$$
v_{i}^{\sharp}=\sum_{k=1}^{n} v_{k} \frac{\partial \Psi_{i}}{\partial z_{k}} .
$$

It follows that

$$
\begin{aligned}
\Gamma_{Q}^{\sharp}(x) & :=\prod_{1 \leq \alpha \neq \beta \leq m}\left(\sum_{i, j=1}^{n} q^{i j} v_{i}^{\sharp}\left(x^{\alpha}\right) v_{j}^{\sharp}\left(x^{\beta}\right)\right) \\
& =\prod_{1 \leq \alpha \neq \beta \leq m}\left(\sum_{i, j, k, l=1}^{n} q^{i j} v_{k}\left(x^{\alpha}\right) v_{l}\left(x^{\beta}\right) \frac{\partial \Psi_{i}}{\partial z_{k}} \frac{\partial \Psi_{j}}{\partial z_{l}}\right) \\
& =\prod_{1 \leq \alpha \neq \beta \leq m}\left(\sum_{k, l=1}^{b} q_{\sharp}^{k l} v_{k}\left(x^{\alpha}\right) v_{l}\left(x^{\beta}\right)\right) \\
& =\Gamma_{Q_{\sharp}}(x)
\end{aligned}
$$

where $Q_{\sharp}$ is the antisymmetric matrix with entries

$$
q_{\sharp}^{k l}=\sum_{i, j=1}^{n} q^{i j} \frac{\partial \Psi_{i}}{\partial z_{k}} \frac{\partial \Psi_{j}}{\partial z_{l}} .
$$

It follows that the vanishing order of $\Gamma_{Q}^{\sharp}$ along $L$ is greater than or equal to $\delta_{y}$. This shows that $\delta_{y} \leq \delta_{y}^{\sharp}$. Applying the same argument in reverse, we get $\delta_{y} \geq \delta_{y}^{\sharp}$. It follows that $\delta_{y}=\delta_{y}^{\sharp}$.

Now let us show that $\delta_{y}$ is independent of the choice of the section $V$ of $\xi$ on $\mathcal{G}$. Let $V^{b}$ be another non-vanishing section and let $\Gamma_{Q}^{b}, \delta_{y}^{b}$ be as defined above using $V^{b}$. Then $V^{b}=h V$ for some non-vanishing holomorphic function $h$ on $\tilde{\mathcal{G}}$. With respect to the coordinate system $\left(z_{1}, \ldots, z_{n}\right)$,

$$
V^{b}=h v_{1} \frac{\partial}{\partial z_{1}}+\cdots+h v_{n} \frac{\partial}{\partial z_{n}},
$$

from which we get

$$
\begin{aligned}
\Gamma_{Q}^{b}(x) & =\prod_{1 \leq \alpha \neq \beta \leq m}\left(\sum_{i, j=1}^{n} q^{i j} h\left(x^{\alpha}\right) v_{i}\left(x^{\alpha}\right) h\left(x^{\beta}\right) v_{j}\left(x^{\beta}\right)\right) \\
& =\Gamma_{Q}(x) \cdot \prod_{1 \leq \alpha \neq \beta \leq m}\left(h\left(x^{\alpha}\right) h\left(x^{\beta}\right)\right) .
\end{aligned}
$$

Since $\prod_{1 \leq \alpha \neq \beta \leq m}\left(h\left(x^{\alpha}\right) h\left(x^{\beta}\right)\right)$ is non-vanishing on $L$, we see that $\delta_{y}=\delta_{y}^{b}$. 
It is easy to see that $\delta_{y}$ does not depend on the choice of the generic point $y \in L$. It will depend only on the choice of an irreducible component $L$ of $E_{\mathcal{M}}$, satisfying the condition $\tau_{L}>1$. We call it the discriminantal order of $L$.

\section{Inverse image webs}

Let $f: X^{\prime} \rightarrow X$ be a generically finite morphism between two projective manifolds. Let $B \subset X$ be the branch divisor and $R \subset X^{\prime}$ be the ramification divisor of $f$. Let $\operatorname{Ker}_{d f} \subset \mathbf{P} T\left(X^{\prime}\right)$ be the projectivization of the kernel of the differential $d f: T\left(X^{\prime}\right) \rightarrow T(X)$. Note that for a generic point $z \in R$, $\operatorname{Ker}_{d f} \cap \mathbf{P} T_{z}(R)=\emptyset$. We denote the projectivization of $d f$ by the same symbol $d f: \mathbf{P} T\left(X^{\prime}\right) \rightarrow \mathbf{P} T(X)$. This is a rational map which is well-defined outside $\operatorname{Ker}_{d f}$.

Proposition 6. Given a generically finite morphism $f: X^{\prime} \rightarrow X$ between two projective manifolds of dimension n, suppose there exists a web $\mathcal{M}$ for $X$ and let $C \subset X$ be a generic member. Then each irreducible component $C^{\prime}$ of $f^{-1}(C)$ has trivial normal bundle.

We start with an elementary lemma.

Lemma 1. Let $f: X^{\prime} \rightarrow X$ be a generically finite morphism between two compact complex manifolds. Let $x \in B$ be a generic point of the branch divisor and $\Delta \subset X$ be a germ of smooth complex analytic curve through $x$ intersecting $B$ transversally. Then $f^{-1}(\Delta)$ is also smooth.

Proof of Lemma 1. Choose a local coordinate system $z_{1}, \ldots, z_{n}$ centered at $x$ and a local coordinate system $w_{1}, \ldots, w_{n}$ centered at a point $x^{\prime} \in f^{-1}(x)$ such that $f$ is given by

$$
z_{1}=w_{1}, \ldots, z_{n-1}=w_{n-1}, \quad z_{n}=w_{n}^{r}
$$

where $r$ is the local sheeting number and $B$ is defined by $z_{n}=0$. Since $\Delta$ is transversal to $B$, it is given by equations of the form

$$
z_{1}=h_{1}\left(z_{n}\right), \ldots, z_{n-1}=h_{n-1}\left(z_{n}\right)
$$

for some convergent power series $h_{1}, \ldots, h_{n-1}$ of one variable $z_{n}$. Thus $f^{-1}(\Delta)$ is defined near $x^{\prime}$ by

$$
w_{1}=h_{1}\left(w_{n}^{r}\right), \ldots, w_{n-1}=h_{n-1}\left(w_{n}^{r}\right),
$$

which is smooth.

Proof of Proposition 6. Let $\mu: \tilde{C} \rightarrow C$ be the normalization. From the genericity of $C$ and Proposition 1, $C$ intersects $B$ transversally. Thus the 
normalization $\mu^{\prime}: \tilde{C}^{\prime} \rightarrow C^{\prime} \subset X^{\prime}$ is an immersion by Lemma 1. Let $\tilde{f}: \tilde{C}^{\prime} \rightarrow$ $\tilde{C}$ be the induced morphism on the normalizations. From the exact sequence

$$
0 \longrightarrow \mathcal{O}_{\tilde{C}}^{n-1} \longrightarrow \mu^{*} T^{*}(X) \longrightarrow T^{*}(\tilde{C}) \longrightarrow 0
$$

we have $n-1$ pointwise independent sections $\omega_{1}, \ldots, \omega_{n-1}$ of $\mu^{*} T^{*}(X)$ annihilating $T(\tilde{C})$. Furthermore, we can assume that any linear combination of $\omega_{1}, \ldots, \omega_{n-1}$ is non-zero on $T_{b}(B)$ for any $b \in C \cap B$. By pulling them back via $\tilde{f}$, we get $n-1$ sections $\omega_{1}^{\prime}, \ldots, \omega_{n-1}^{\prime}$ of $\mu^{\prime *} T^{*}\left(C^{\prime}\right)$ on $\tilde{C}^{\prime}$ which are pointwise linearly independent at points different from $\mu^{\prime-1}(R)$. From the genericity of $C$, we may assume that $\left.f\right|_{R}$ is unramified at $C^{\prime} \cap R$. Thus for any $b^{\prime} \in C^{\prime} \cap R$ and $b=f\left(b^{\prime}\right) \in C \cap B$, the differential $d f: T_{b^{\prime}}(R) \rightarrow T_{b}(B)$ is an isomorphism. It follows that any linear combination of $\omega_{1}^{\prime}, \ldots, \omega_{n-1}^{\prime}$ is non-zero on $T_{b^{\prime}}(R)$. This implies that $\omega_{1}^{\prime}, \ldots, \omega_{n-1}^{\prime}$ give $n-1$ pointwise independent sections of $\mu^{\prime *} T^{*}\left(X^{\prime}\right)$ annihilating $T\left(\tilde{C}^{\prime}\right)$, showing that $C^{\prime}$ has trivial normal bundle.

Remark. An important case where Proposition 6 will be applied is when both $X$ and $X^{\prime}$ are Fano manifolds (Section 6). Note that any curve with trivial normal bundle on a Fano manifold must be a rational curve and immersed rational curves through generic points have semi-positive normal bundle. In this case, the proof of Proposition 6 gets slightly simpler. In fact, since the conormal bundle of $C$ is trivial, the conormal bundle of $C^{\prime}$, which is immersed by Lemma 1, has non-negative degree because it is generated by global sections at generic points by pulling back the global sections of the conormal bundle of $C$. It follows that $K_{C^{\prime}}^{-1} \geq\left. K_{X^{\prime}}^{-1}\right|_{C^{\prime}}>0$ since $X^{\prime}$ is Fano. Thus $C^{\prime}$ is a rational curve. From the genericity of $C$, the normal bundle of $C^{\prime}$ is semi-positive. Since the conormal bundle is generated by global sections at a generic point, $C^{\prime}$ has trivial normal bundle.

From Proposition 6, the components of $f^{-1}(C)$ for various choices of $[C] \in$ $\mathcal{M}$ define a web $\mathcal{M}^{\prime}$ on $X^{\prime}$. This web $\mathcal{M}^{\prime}$ is called the inverse image web of $\mathcal{M}$ under $f$. Let $\psi^{\prime}: F^{\prime} \rightarrow \mathcal{M}^{\prime}$ and $\phi^{\prime}: F^{\prime} \rightarrow X^{\prime}$ be the associated universal family morphisms. As before, we assume the normality of $F^{\prime}$ and $\mathcal{M}^{\prime}$. Let $\Phi^{\prime}: F^{\prime} \rightarrow \mathbf{P} T\left(X^{\prime}\right)$ be the tangent map and $\mathcal{C}^{\prime} \subset \mathbf{P} T\left(X^{\prime}\right)$ be the variety of $\mathcal{M}^{\prime}$-tangents. Then $d f\left(\mathcal{C}^{\prime}\right)$, the strict image of $\mathcal{C}^{\prime}$ under the rational map $d f: \mathbf{P} T\left(X^{\prime}\right) \rightarrow \mathbf{P} T(X)$, is exactly $\mathcal{C}$. The union of the components of $d f^{-1}(\mathcal{C})$ which are dominant over $X^{\prime}$ is exactly $\mathcal{C}^{\prime}$. The degree of $\mathcal{M}^{\prime}$ is the same as that of $\mathcal{M}$.

Proposition 7. Let $f: X^{\prime} \rightarrow X$ be a generically finite morphism between two projective manifolds. Suppose there exists a web $\mathcal{M}$ on $X$ and $\mathcal{M}^{\prime}$ is the inverse image web on $X^{\prime}$. Then $f^{-1}\left(E_{\mathcal{M}}\right) \subset E_{\mathcal{M}^{\prime}}$. 
Proof. Let $L$ be an irreducible component of $E_{\mathcal{M}}$. Suppose for a generic point $y$ of $L$, there exists $y^{\prime} \in f^{-1}(y)$ with $y^{\prime} \notin E_{\mathcal{M}^{\prime}}$. Let $d$ be the degree of $\mathcal{M}$ and $\mathcal{M}^{\prime}$. Since $\pi^{\prime}: \mathcal{C}^{\prime} \rightarrow X^{\prime}$ is unramified at $y^{\prime}$, there exist $d$ distinct germs $C_{1}^{\prime}, \ldots, C_{d}^{\prime}$ of smooth curves through $y^{\prime}$ with distinct tangent vectors at $y^{\prime}$ which are germs of members of $\mathcal{M}^{\prime}$.

If $y^{\prime}$ is not in $R, C_{1}:=f\left(C_{1}^{\prime}\right), \ldots, C_{d}:=f\left(C_{d}^{\prime}\right)$ give $d$ distinct germs of smooth curves through $y$ with distinct tangents which are germs of members of $\mathcal{M}$, a contradiction to $y \in E_{\mathcal{M}}$.

Suppose $y^{\prime} \in R$. From the genericity of $y$, this means that the component of $R$ containing $y$ is sent to a component of $E_{\mathcal{M}}$ by $f$. We may assume that $y^{\prime}$ is a smooth point of $R$ and $d f_{y^{\prime}}: T_{y^{\prime}}(R) \rightarrow T_{y}(L)$ is an isomorphism. We claim that there is at most one germ among $C_{1}^{\prime}, \ldots, C_{d}^{\prime}$ which is not contained in $R$. In fact, suppose $C_{1}^{\prime}$ and $C_{2}^{\prime}$ do not lie on $R$. Then $f\left(C_{1}^{\prime}\right)$ and $f\left(C_{2}^{\prime}\right)$ are germs of members of $\mathcal{M}$ which do not lie on $L$. From Proposition 1, $f\left(C_{1}^{\prime}\right)$ and $f\left(C_{2}^{\prime}\right)$ are transversal to $L$ at $y$. This implies that $C_{1}^{\prime}$ and $C_{2}^{\prime}$ are tangent to the kernel of $d f$ at $y^{\prime}$; however, they have distinct tangent vectors at $y^{\prime}$, a contradiction.

Suppose all of $C_{1}^{\prime}, \ldots, C_{d}^{\prime}$ are contained in $R$. Since $d f_{y^{\prime}}: T_{y^{\prime}}(R) \rightarrow T_{y}(L)$ is an isomorphism, $f\left(C_{1}^{\prime}\right), \ldots, f\left(C_{d}^{\prime}\right)$ give $d$ distinct germs of smooth curves through $y$ with distinct tangents which are germs of members of $\mathcal{M}$. A contradiction to $y \in L$.

Suppose $C_{1}^{\prime}$ is not contained in $R$ and all of $C_{2}^{\prime}, \ldots, C_{d}^{\prime}$ are contained in $R$. Then $f\left(C_{1}^{\prime}\right)$ must be transversal to $L$ by Proposition 1 , while $f\left(C_{2}^{\prime}\right), \ldots, f\left(C_{d}^{\prime}\right)$ give $d-1$ distinct germs of smooth curves through $y$ with distinct tangent vectors at $y$ which are germs of members of $\mathcal{M}$, a contradiction to $y \in L$ again.

Proposition 8. In the situation of Proposition 7, let $L$ be a component of $E_{\mathcal{M}}$ and $L^{\prime}$ be a component of $f^{-1}(L) \subset E_{\mathcal{M}^{\prime}}$. Then $\tau_{L}=\tau_{L^{\prime}}$.

Proof. Let $y \in L$ be a generic point and $y^{\prime} \in f^{-1}(y) \subset L^{\prime}$. Let $U$ be an isolating neighborhood of $y$ with respect to $\pi: \mathcal{C} \rightarrow X$ and $U^{\prime}$ be an isolating neighborhood of $y^{\prime}$ with respect to $\pi^{\prime}: \mathcal{C}^{\prime} \rightarrow X^{\prime}$. We may assume that $U=f\left(U^{\prime}\right)$. Let $\mathcal{G}$ (resp. $\mathcal{G}^{\prime}$ ) be the union of components of $\left.\mathcal{C}\right|_{U}$ (resp. $\left.\left.\mathcal{C}^{\prime}\right|_{U^{\prime}}\right)$ which intersect $\mathbf{P} T\left(E_{\mathcal{M}}\right)$ (resp. $\mathbf{P} T\left(E_{\mathcal{M}^{\prime}}\right)$ ). Recall that $\tau_{L}$ is the degree of $\pi$ on $\mathcal{G}$ and $\tau_{L^{\prime}}$ is the degree of $\pi^{\prime}$ on $\mathcal{G}^{\prime}$.

The strict image of $\left.\mathcal{C}^{\prime}\right|_{U^{\prime}}$ under $\left.d f\right|_{U^{\prime}}$ is contained in $\left.\mathcal{C}\right|_{U}$. Since $d f_{u^{\prime}}$ : $\mathbf{P} T_{u^{\prime}}\left(X^{\prime}\right) \rightarrow \mathbf{P} T_{u}(X)$ is an isomorphism for a generic $u^{\prime} \in U^{\prime}$ with $u=f\left(u^{\prime}\right)$, the degree of $\pi$ on the strict image $d f\left(\left.\mathcal{C}^{\prime}\right|_{U^{\prime}}\right)$ is $d$, the degree of $\mathcal{M}$ and $\mathcal{M}^{\prime}$. It follows that $d f\left(\left.\mathcal{C}^{\prime}\right|_{U^{\prime}}\right)=\left.\mathcal{C}\right|_{U}$. 
The genericity of $y^{\prime}$ implies that $\mathbf{P} T_{y}\left(L^{\prime}\right) \cap K e r_{d f}=\emptyset$. Thus the rational map $d f: \mathbf{P} T\left(U^{\prime}\right) \rightarrow \mathbf{P} T(U)$ is a well-defined morphism on $\mathcal{G}^{\prime}$. Then it is clear that $d f\left(\mathcal{G}^{\prime}\right) \subset \mathcal{G}$ and $\tau_{L^{\prime}} \leq \tau_{L}$.

Let $\mathcal{A}$ (resp. $\mathcal{A}^{\prime}$ ) be the union of components of $\left.\mathcal{C}\right|_{U}$ (resp. $\left.\mathcal{C}^{\prime}\right|_{U^{\prime}}$ ) which are disjoint from $\mathbf{P} T(L)$ (resp. $\left.\mathbf{P} T\left(L^{\prime}\right)\right)$ so that

$$
\begin{aligned}
\left.\mathcal{C}\right|_{U} & =\mathcal{G} \cup \mathcal{A}, \\
\left.\mathcal{C}^{\prime}\right|_{U^{\prime}} & =\mathcal{G}^{\prime} \cup \mathcal{A}^{\prime} .
\end{aligned}
$$

From Proposition $3, \mathcal{A}\left(\right.$ resp. $\left.\mathcal{A}^{\prime}\right)$ is disjoint from $\Phi(\mathcal{R})\left(\right.$ resp. $\Phi^{\prime}\left(\mathcal{R}^{\prime}\right)$ ) where $\mathcal{R}$ (resp. $\mathcal{R}^{\prime}$ ) is the ramification divisor of $\phi: F \rightarrow X$ (resp. $\phi^{\prime}: F^{\prime} \rightarrow X^{\prime}$ ). By shrinking $U$ (resp. $U^{\prime}$ ), one can assume that $\phi$ (resp. $\phi^{\prime}$ ) is locally one-to-one over $U$ (resp. $U^{\prime}$ ) outside $\mathcal{R}$ (resp. $\mathcal{R}^{\prime}$ ) and $\Phi$ (resp. $\Phi^{\prime}$ ) is a normalization map over $U$ (resp. $U^{\prime}$ ) by Proposition 2. Thus $\pi$ (resp. $\pi^{\prime}$ ) is 1-to-1 on each component of $\mathcal{A}$ (resp. $\left.\mathcal{A}^{\prime}\right)$.

We claim that the strict image of $\mathcal{A}^{\prime}$ under $d f$ is contained in $\mathcal{A}$. This is obvious if $y^{\prime} \notin R$, the ramification divisor of $f$. Thus we may assume that $L^{\prime} \subset R$. Since $\pi^{\prime}$ is 1 -to- 1 on each component $A^{\prime}$ of $\mathcal{A}^{\prime}$, we may assume that $A^{\prime}$ defines a foliation by smooth curves on $U^{\prime}$. Since $A^{\prime}$ is disjoint from $\mathbf{P} T\left(L^{\prime}\right)$, the leaves of this foliation are transversal to $L^{\prime}$. Each leaf of this foliation is part of a member of $\mathcal{M}^{\prime}$. Thus from the genericity of $y^{\prime}$, we may assume that the leaves are parts of generic members of $\mathcal{M}^{\prime}$ and their images under $f$ are parts of generic members of $\mathcal{M}$. From Proposition 1, the images of leaves of the foliation on $U^{\prime}$ are sent to germs of curves transversal to $L$. Thus by shrinking $U^{\prime}$ if necessary, we may assume that the image of this foliation under $f$ defines a foliation by smooth curves in a neighborhood of $y$ whose leaves are transversal to $L$. This foliation defines the strict image $d f\left(A^{\prime}\right)$ which contains a unique point in $\mathcal{C} \cap \pi^{-1}(y)$ corresponding to the tangent vector of the leaf through $y$. Since the leaf is transversal to $L, d f\left(A^{\prime}\right)$ is disjoint from $\mathbf{P} T(L)$. Thus $d f\left(A^{\prime}\right)$ is an irreducible component of $\mathcal{A}$.

Let $e$ (resp. $e^{\prime}$ ) be the degree of $\pi$ (resp. $\left.\pi^{\prime}\right)$ on $\mathcal{A}$ (resp. $\mathcal{A}^{\prime}$ ). Since $d f_{u^{\prime}}$ for a generic $u^{\prime} \in U^{\prime}$ is an isomorphism, $d f\left(\mathcal{A}^{\prime}\right) \subset \mathcal{A}$ implies that $e \geq e^{\prime}$. Since $d=\tau_{L}+e=\tau_{L^{\prime}}+e^{\prime}$, we see that $\tau_{L^{\prime}} \geq \tau_{L}$ and we are done.

Remark. One cannot rule out the possibility that an irreducible component of $\left.\mathcal{C}\right|_{U}$ is pulled back to a reducible set in $\left.\mathcal{C}^{\prime}\right|_{U}$. This point causes some complications in the statements and proofs of some results of this paper. For example, we are not able to extend Proposition 7 to a statement like $f^{-1}\left(D_{\mathcal{M}}\right) \subset D_{\mathcal{M}^{\prime}}$. 


\section{Discriminantal orders of inverse image webs}

We will continue to use the notation in the previous section. We are given a generically finite morphism $f: X^{\prime} \rightarrow X$ between two projective manifolds of dimension $n$, a web $\mathcal{M}$ on $X$ and the inverse image web $\mathcal{M}^{\prime}$. Let $M$ be a component of $D_{\mathcal{M}}$ and $L^{\prime}$ be a component of $f^{-1}(M) \subset E_{\mathcal{M}^{\prime}}$ given by Proposition 7. By Proposition 4 and Proposition 8, $1<\tau_{M}=\tau_{L^{\prime}}$. Thus the discriminantal orders $\delta_{M}$ and $\delta_{L}$ are well-defined. If $L^{\prime}$ is not contained in the ramification divisor $R$ of $f$, then $\delta_{L^{\prime}}=\delta_{M}$. When $L^{\prime}$ is a component of $R$, we have the following result.

Proposition 9. Given a generically finite morphism $f: X^{\prime} \rightarrow X$, a web $\mathcal{M}$ and its inverse image $\mathcal{M}^{\prime}$ as above, let $L^{\prime}$ be a component of $E_{\mathcal{M}^{\prime}}$ which is at the same time a component of the ramification divisor $R$ of $f$ and satisfies $M:=f\left(L^{\prime}\right) \subset D_{\mathcal{M}}$. Write $r>1$ for the local sheeting number of $f$ at a generic point of $L^{\prime}$ and let $m>1$ be the tangential sheeting number of $L^{\prime}$ and M. Then $r \leq m \delta_{L^{\prime}}$.

Proof. We may assume that $r>m$. Let $y^{\prime} \in L^{\prime}$ be a generic point and set $y=f\left(y^{\prime}\right) \in M$. We can choose a coordinate system $\left(w_{1}, \ldots, w_{n}\right)$ on an isolating neighborhood $U^{\prime}$ of $y^{\prime}$ with respect to $\mathcal{C}^{\prime}$ and a coordinate system $\left(z_{1}, \ldots, z_{n}\right)$ on an isolating neighborhood $U=f\left(U^{\prime}\right)$ of $y$ with respect to $\mathcal{C}$, so that $f$ is given by

$$
z_{1}=w_{1}, \ldots, z_{n-1}=w_{n-1}, \quad z_{n}=w_{n}^{r} .
$$

Note that $z_{n}=0$ defines $M \cap U$ and $w_{n}=0$ defines $L^{\prime} \cap U^{\prime}$. Let $\left.\mathcal{G} \subset \mathcal{C}\right|_{U}$ be the union of components intersecting $\mathbf{P} T(M)$ and let $\left.\mathcal{G}^{\prime} \subset \mathcal{C}^{\prime}\right|_{U^{\prime}}$ be the union of components intersecting $\mathbf{P} T\left(L^{\prime}\right)$. By definition, the tangential sheeting number $m$ is the degree of $\pi^{\prime}$ on $\mathcal{G}^{\prime}$, which is equal to the degree of $\pi$ on $\mathcal{G}$, by Proposition 8. Let $\xi$ be the tautological line bundle of $\mathbf{P} T(X)$ restricted to the normalization $\tilde{\mathcal{G}}$ of $\mathcal{G}$. By shrinking $\mathcal{U}$, we assume that $\xi$ is trivial on $\tilde{\mathcal{G}}$ as in Section 2. Let

$$
V=v_{1} \frac{\partial}{\partial z_{1}}+\cdots+v_{n} \frac{\partial}{\partial z_{n}}
$$

be a section of $\xi$ on $\tilde{\mathcal{G}}$ as in Section 2. Since $\mathcal{G} \cap \pi^{-1}(y) \subset \mathbf{P} T_{y}(M), v_{n}$ vanishes over $z_{n}=0$ and one of $v_{1}, \ldots, v_{n-1}$ is non-vanishing at points over $U \cap M$. Consider

$$
f^{*} V:=d f^{*} v_{1} \frac{\partial}{\partial w_{1}}+\cdots+d f^{*} v_{n-1} \frac{\partial}{\partial w_{n-1}}+\frac{d f^{*} v_{n}}{r \cdot w_{n}^{r-1}} \frac{\partial}{\partial w_{n}},
$$

where $d f^{*} v_{i}$ is the pull-back of the holomorphic function $v_{i}$ on $\tilde{\mathcal{G}}$ by the holomorphic map $d f: \tilde{\mathcal{G}}^{\prime} \rightarrow \tilde{\mathcal{G}}$ which is the lifting of $d f: \mathcal{G}^{\prime} \rightarrow \mathcal{G}$ to the normalizations. $f^{*} V$ is a meromorphic section of the tautological bundle $\xi^{\prime}$ of $\mathbf{P} T\left(X^{\prime}\right)$ over $\tilde{\mathcal{G}}^{\prime}$. 
We claim that the meromorphic function $\frac{d f^{*} v_{n}}{w_{n}^{r-1}}$ on $\tilde{\mathcal{G}}^{\prime}$ must be holomorphic. Suppose not. Since we are interested in a generic point $y^{\prime} \in L^{\prime}$, we may assume that $\tilde{\mathcal{G}}^{\prime}$ is smooth and a generic point $a$ of the polar divisor of $\frac{d f^{*} v_{n}}{w_{n}^{r-1}}$ lies over $\mathcal{G}^{\prime} \cap \mathbf{P} T\left(L^{\prime}\right)$. Let $h$ be a holomorphic function on $\tilde{\mathcal{G}}^{\prime}$ defining the polar divisor so that $h \frac{d f^{*} v_{n}}{w_{n}^{r-1}}$ is non-vanishing at $a$. Thus $h f^{*} V$ is a non-vanishing section of $\xi^{\prime}$ in a neighborhood of $a$ in $\tilde{\mathcal{G}}^{\prime}$. However, at a point of $h=0$,

$$
h f^{*} V=h \frac{d f^{*} v_{n}}{w_{n}^{r-1}} \frac{\partial}{\partial w_{n}}
$$

corresponds to a tangent vector transversal to $L^{\prime}$. This is a contradiction to the fact that $\mathcal{G}^{\prime} \cap \pi^{-1}\left(y^{\prime}\right) \subset \mathbf{P} T_{y^{\prime}}\left(L^{\prime}\right)$.

Since one of $d f^{*} v_{1}, \ldots, d f^{*} v_{n-1}$ is non-vanishing on $\tilde{\mathcal{G}}^{\prime}$, we see that $f^{*} V$ is a non-vanishing section of $\xi^{\prime}$ over $\tilde{\mathcal{G}}^{\prime}$. Thus we can compute the discriminantal order $\delta_{L^{\prime}}$ using $f^{*} V$. As in Section 2, for a given $Q=\left(q^{i j}\right) \in \mathbf{o}(n, \mathbf{C})$, let

$$
\begin{aligned}
\Gamma_{Q}(x) & =\prod_{1 \leq \alpha \neq \beta \leq m}\left(\sum_{i, j=1}^{n} q^{i j} v_{i}\left(x^{\alpha}\right) v_{j}\left(x^{\beta}\right)\right), \\
\Gamma_{Q}^{\prime}\left(x^{\prime}\right) & =\prod_{1 \leq \alpha \neq \beta \leq m}\left(\sum_{i, j=1}^{n} q^{i j} v_{i}^{\prime}\left(u^{\alpha}\right) v_{j}^{\prime}\left(u^{\beta}\right)\right),
\end{aligned}
$$

where $\left\{u^{1}, \ldots, u^{m}\right\}$ are the points of $\mathcal{G}^{\prime}$ over a generic point $u \in U^{\prime}$, $\left\{x^{1}=d f_{u}\left(u^{1}\right), \ldots, x^{m}=d f_{u}\left(u^{m}\right)\right\}$ are the points of $\mathcal{G}$ over $x=f(u)$, and

$$
v_{1}^{\prime}=d f^{*} v_{1}, \ldots, v_{n-1}^{\prime}=d f^{*} v_{n-1}, \quad v_{n}^{\prime}=\frac{d f^{*} v_{n}}{r \cdot w_{n}^{r-1}} .
$$

For each $\mu=(\alpha, \beta) \in I$, we define

$$
\begin{aligned}
& \Lambda_{\mu}:=\sum_{i, j=1}^{n-1} q^{i j} v_{i}\left(x^{\alpha}\right) \cdot v_{j}\left(x^{\beta}\right), \\
& \Lambda_{\mu}^{\prime}:=\sum_{i, j=1}^{n-1} q^{i j} d f^{*} v_{i}\left(u^{\alpha}\right) \cdot d f^{*} v_{j}\left(u^{\beta}\right), \\
& \Omega_{\mu}:=\sum_{i=1}^{n-1} q^{i n} v_{i}\left(x^{\alpha}\right) v_{n}\left(x^{\beta}\right)+\sum_{i=1}^{n-1} q^{n i} v_{i}\left(x^{\beta}\right) v_{n}\left(x^{\alpha}\right), \\
& \Omega_{\mu}^{\prime}:=\sum_{i=1}^{n-1} q^{i n} d f^{*} v_{i}\left(u^{\alpha}\right) \frac{d f^{*} v_{n}\left(u^{\beta}\right)}{r \cdot w_{n}^{r-1}}+\sum_{i=1}^{n-1} q^{n i} d f^{*} v_{i}\left(u^{\beta}\right) \frac{d f^{*} v_{n}\left(u^{\alpha}\right)}{r \cdot w_{n}^{r-1}},
\end{aligned}
$$

so that

$$
\Gamma_{Q}^{\prime}(u)=\prod_{\mu \in I}\left(\Lambda_{\mu}^{\prime}+\Omega_{\mu}^{\prime}\right)
$$


To prove Proposition 9, it suffices to show that the vanishing order of $\Gamma_{Q}^{\prime}$ on $w_{n}=0$ is $\geq \frac{r}{m}$.

Consider the holomorphic arc $\rho(t) \in U, t \in \Delta_{\epsilon}$ defined by $z_{1}=\cdots=$ $z_{n-1}=0, z_{n}=t$, and the holomorphic arc $\rho^{\prime}(s) \in U^{\prime}, s \in \Delta_{\epsilon^{\prime}}$ defined by $w_{1}=\cdots=w_{n-1}=0, w_{n}=s . f$ restricted to these arcs is given by $t=s^{r}$. By restricting to these holomorphic arcs, we regard $\Lambda_{\mu}$ and $\Omega_{\mu}$ as multi-valued functions of the variable $t$ and $\Lambda_{\mu}^{\prime}$ and $\Omega_{\mu}^{\prime}$ as multi-valued functions of the variable $s$. By Puiseux expansion, we can consider the fractional vanishing orders $\lambda_{\mu}$ (resp. $\omega_{\mu}$ ) of $\Lambda_{\mu}$ (resp. $\Omega_{\mu}$ ) in $t$ and the fractional vanishing orders $\lambda_{\mu}^{\prime}\left(\right.$ resp. $\left.\omega_{\mu}^{\prime}\right)$ of $\Lambda_{\mu}^{\prime}\left(\right.$ resp. $\left.\Omega_{\mu}^{\prime}\right)$ in $s$. Since

$$
\begin{aligned}
\Lambda_{\mu}^{\prime} & =d f^{*} \Lambda_{\mu}, \\
\Omega_{\mu}^{\prime} & =\frac{1}{r \cdot w_{n}^{r-1}} d f^{*} \Omega_{\mu},
\end{aligned}
$$

we have $\lambda_{\mu}^{\prime}=r \lambda_{\mu}$ and $\omega_{\mu}^{\prime}=r \omega_{\mu}-(r-1)=\left(\omega_{\mu}-1\right) r+1$.

From $y \in M$, there exists an irreducible component of $\mathcal{G}^{o}$ of $\mathcal{G}$ which is generically $k$-to-1 over $\pi\left(\mathcal{G}^{\circ}\right)$ for some $k>1$. Regard $V$ as a non-vanishing section of $\xi$ over $\mathcal{G}^{o}$ and $v_{i}$ as holomorphic functions on the normalization $\tilde{\mathcal{G}}^{o}$. By Puiseux expansion, there exist convergent power series $g_{1}(\tau), \ldots, g_{n}(\tau)$ of a variable $\tau$, so that

$$
\left.v_{1}\right|_{\rho\left(\Delta_{\epsilon}\right)}=g_{1}\left(t^{\frac{1}{k}}\right), \ldots,\left.v_{n}\right|_{\rho\left(\Delta_{\epsilon}\right)}=g_{n}\left(t^{\frac{1}{k}}\right) .
$$

Let $g$ be the order of $g_{n}(\tau)$ in $\tau$. Then

$$
\left.\frac{d f^{*} v_{n}}{r \cdot w_{n}^{r-1}}\right|_{\rho^{\prime}\left(\Delta_{\epsilon^{\prime}}\right)}=\frac{g_{n}\left(s^{\frac{r}{k}}\right)}{r \cdot s^{r-1}}
$$

has vanishing order $\frac{g r}{k}-r+1$ in $s$, which must be non-negative. So we get $g \geq \frac{k(r-1)}{r}$. From the assumption $r>m$, we see $g \geq k$.

Choose $\nu=(\alpha, \beta) \in I$ so that both $x^{\alpha}$ and $x^{\beta}$ lie on $\mathcal{G}^{o}$. Then both $\lambda_{\nu}$ and $\omega_{\nu}$ are $\geq \frac{1}{k}$. For $\zeta=e^{\frac{2 \pi \sqrt{-1}}{k}}$, we can write

$$
\left.\Omega_{\nu}\right|_{\rho\left(\Delta_{\epsilon}\right)}=\sum_{i=1}^{n-1} q^{i n}\left(g_{i}\left(\zeta^{\alpha} t^{\frac{1}{k}}\right) \cdot g_{n}\left(\zeta^{\beta} t^{\frac{1}{k}}\right)-g_{i}\left(\zeta^{\beta} t^{\frac{1}{k}}\right) \cdot g_{n}\left(\zeta^{\alpha} t^{\frac{1}{k}}\right)\right) .
$$

We claim that this has vanishing order $\geq k+1$ in $t^{\frac{1}{k}}$. The claim is obvious if $g>k$. So assume that $g=k$. Suppose $g_{i}$ has no constant term. Then it is obvious that the $i$-th term of $\Omega_{\nu}$ has vanishing order $\geq g+1=k+1$ in $t^{\frac{1}{k}}$. Suppose $g_{i}$ has a non-zero constant term. Then the lowest order term of $g_{i}\left(\zeta^{\alpha} t^{\frac{1}{k}}\right) \cdot g_{n}\left(\zeta^{\beta} t^{\frac{1}{k}}\right)$ cancels that of $g_{i}\left(\zeta^{\beta} t^{\frac{1}{k}}\right) \cdot g_{n}\left(\zeta^{\alpha} t^{\frac{1}{k}}\right)$ because $\zeta^{g}=1$. Thus in all cases, the vanishing order of $\Omega_{\nu}$ is $\geq k+1$ in $t^{\frac{1}{k}}$. In other words, 
$\omega_{\nu} \geq 1+\frac{1}{k}$. We conclude that $\lambda_{\nu}^{\prime}=r \lambda_{\nu} \geq \frac{r}{k}$ and $\omega_{\nu}^{\prime}=\left(\omega_{\nu}-1\right) r+1 \geq \frac{r}{k}+1$. Thus the vanishing order of

$$
\Gamma_{Q}^{\prime}(u)=\prod_{\mu \in I}\left(\Lambda_{\mu}^{\prime}+\Omega_{\mu}^{\prime}\right)
$$

is $\geq \min \left(\lambda_{\nu}^{\prime}, \omega_{\nu}^{\prime}\right) \geq \min \left(\frac{r}{k}, \frac{r}{k}+1\right) \geq \frac{r}{m}$.

\section{Deformation rigidity of generically finite morphisms}

Let $X$ be a projective manifold and $C$ be a rational curve with trivial normal bundle on $X$. Since $H^{1}\left(\mathbf{P}_{1}, \mathcal{O}^{n-1}\right)=0$, we can deform $C$ as rational curves to cover an open subset of $X$. Thus the existence of a rational curve with trivial normal bundle is equivalent to the existence of a web of rational curves. As mentioned in the introduction, this is also equivalent to the existence of a family of rational curves covering $X$ which have degree 2 with respect to $K_{X}^{-1}$. This property is preserved under deformation:

Proposition 10. Let $\chi: \mathcal{X} \rightarrow \Delta$ be a regular family of projective manifolds. Suppose $X_{0}:=\chi^{-1}(0)$ has a web $\mathcal{M}_{0}$ of rational curves; then, for some $\epsilon>0, X_{t}:=\chi^{-1}(t)$ has a web $\mathcal{M}_{t}$ of rational curves for all $0<|t|<\epsilon$ so that $\mathcal{M}_{t}$ forms a flat family over $0<|t|<\epsilon$ and members of $\mathcal{M}_{0}$ are limits of members of $\mathcal{M}_{t}$.

In the statement of Proposition 10, we do not exclude the possibility that the limit of $\mathcal{M}_{t}$ at $t=0$ has other components different from $\mathcal{M}_{0}$.

Proof. Let $C$ be a generic member of a component of $\mathcal{M}_{0}$. Since $C$ has trivial normal bundle in $X_{0}$, it has trivial normal bundle in the complex manifold $\mathcal{X}$. Thus $C$ can be deformed as rational curves to cover an open subset of $\mathcal{X}$. This also follows from Kodaira stability $([\overline{\mathrm{Kd}}])$ since $H^{1}\left(\mathbf{P}_{1}, N_{C \subset X_{0}}\right)=0$ for the normal bundle $N_{C \subset X_{0}} \cong \mathcal{O}_{C}^{n-1}$. Let $\mathcal{D}$ be the irreducible component of the normalized Douady-Hilbert scheme of rational curves in $\mathcal{X}$ containing the point $[C]$ corresponding to $C$. Then $\mathcal{D}$ is smooth at $[C]$ and the natural projection $\mathcal{D} \rightarrow \Delta$ is of maximal rank at $[C]$. Let $\mathcal{D}_{t}$ be the the fiber of $\mathcal{D} \rightarrow \Delta$ at $t \in \Delta$. Since all members of $\mathcal{D}_{t}$ have degree 2 with respect to $K_{X_{t}}^{-1}$, a component of $\mathcal{D}_{t}$ whose members cover $X_{t}$ is an irreducible web on $X_{t}$. Let $\mathcal{D}_{t}^{o}$ be the union of the components of $\mathcal{D}_{t}$ whose members cover $X_{t}$. Then let $\mathcal{M}_{t}$ be the union of all $\mathcal{D}_{t}^{o}$ which arises as we choose $C$ from different components of $\mathcal{M}_{0}$. Clearly, $\left\{\mathcal{M}_{t}, 0<|t|<\epsilon\right\}$ form a flat family for a suitable choice of $\epsilon$. 
Proposition 11. Let $\chi: \mathcal{X} \rightarrow \Delta$ be as in Proposition 10 and $\mathcal{C}_{t} \subset \mathbf{P} T\left(X_{t}\right)$ be the variety of $\mathcal{M}_{t}$-tangents. For a given projective manifold $X^{\prime}$, let $\mathcal{X}^{\prime}:=$ $X^{\prime} \times \Delta$. Suppose there exists a morphism $f: \mathcal{X}^{\prime} \rightarrow \mathcal{X}$ respecting the projections to $\Delta$, such that $f_{t}: X^{\prime} \rightarrow X_{t}$ is generically finite for each $t \in \Delta$. Then there exists a subvariety $\mathcal{C}^{\prime} \subset \mathbf{P} T\left(X^{\prime}\right)$ so that the union of components of $d f_{t}^{-1}\left(\mathcal{C}_{t}\right)$ dominant over $X^{\prime}$ is $\mathcal{C}^{\prime}$ whenever $0<|t|<\epsilon$; the union of components of $d f_{0}^{-1}\left(\mathcal{C}_{0}\right)$ dominant over $X^{\prime}$ is contained in $\mathcal{C}^{\prime}$.

Proof. Let $\mathcal{C}_{t}^{\prime} \subset \mathbf{P} T\left(X^{\prime}\right)$ be the union of components of $d f_{t}^{-1}\left(\mathcal{C}_{t}\right)$ dominant over $X^{\prime} . \mathcal{C}_{t}^{\prime}$ is a flat family for $0<|t|<\epsilon . \mathcal{C}_{0}^{\prime}$ is contained in the closure of the union of $\left\{\mathcal{C}_{t}^{\prime}, 0<|t|<\epsilon\right\}$. From Section $3,\left\{\mathcal{C}_{t}^{\prime}, 0<|t|<\epsilon\right\}$ gives a flat family of webs on $X^{\prime}$. Since there are only countably many webs on $X^{\prime}, \mathcal{C}_{t}^{\prime}$ must be independent of $t$. Just set $\mathcal{C}^{\prime}=\mathcal{C}_{t}^{\prime}$ for any $t, 0<|t|<\epsilon$.

Note that any web on a Fano manifold is a web of rational curves. In the previous sections, we made the assumption that the discriminantal divisor of a web is non-empty. This is always the case for webs on a Fano manifold of Picard number 1:

Proposition 12. Let $X$ be a Fano manifold of Picard number 1 and $\mathcal{M}$ be a web. Let $\psi: F \rightarrow \mathcal{M}$ and $\phi: F \rightarrow X$ be the universal family morphisms. Then $\phi$ is not birational on any component of $F$. Consequently, the discriminantal divisor $D_{\mathcal{M}} \subset X$ is non-empty.

Proof. We may assume that $\mathcal{M}$ and $F$ are irreducible. Recall that $\phi$ is unramified in an analytic neighborhood of a generic fiber $\psi^{-1}(v), v \in \mathcal{M}$. Choose a hypersurface $H \subset \mathcal{M}$ disjoint from $v$ such that $\phi\left(\psi^{-1}(H)\right)$ is a hypersurface in $X$. If $\phi$ is birational, the curve $\phi\left(\psi^{-1}(v)\right)$ is disjoint from the hypersurface $\phi\left(\psi^{-1}(H)\right)$ in $X$, a contradiction to the fact that $X$ is of Picard number 1.

Now let us go to the situation of Theorem 1 :

Proposition 13. Let $\chi: \mathcal{X} \rightarrow \Delta:=\{t \in \mathbf{C},|t|<1\}$ be a regular family of Fano manifolds of Picard number 1 so that $X_{0}=\chi^{-1}(0)$ has a web $\mathcal{M}_{0}$ of rational curves. By Proposition 10, we have a web $\mathcal{M}_{t}$ on $X_{t}$ for $0<|t|<$ $\epsilon$. Let $Z_{t} \subset X_{t}$ be the subvariety of codimension $>1$ so that the universal family morphism $\phi_{t}: F_{t} \rightarrow X_{t}$ associated to the web $\mathcal{M}_{t}$ is unramified over $X_{t}-D_{\mathcal{M}_{t}}-Z_{t}$. Given $f: \mathcal{X}^{\prime} \rightarrow \mathcal{X}$ as in Proposition 11, let $\mathcal{M}^{\prime}$ be the web defined by $\mathcal{C}^{\prime}$ on $X^{\prime}$. For a generic member $C \subset X_{0}$ of $\mathcal{M}_{0}$ and any irreducible component $C^{\prime}$ of $f_{0}^{-1}(C)$, the following holds:

(i) $C^{\prime}$ intersects $E_{\mathcal{M}^{\prime}}$ transversally;

(ii) $C_{t}:=f_{t}\left(C^{\prime}\right)$ is a member of $\mathcal{M}_{t}$ having trivial normal bundle, is not contained in $D_{\mathcal{M}_{t}}$ and is disjoint from $Z_{t}$ for all $0<|t|<\epsilon$.

Proof. This is a direct consequence of Proposition 1 and $D_{\mathcal{M}_{t}} \subset f_{t}\left(E_{\mathcal{M}^{\prime}}\right)$ which follows from Proposition 7. 
Proposition 14. Under the assumptions of Proposition 13, for each fixed $t \in \Delta$, the intersection $C_{t} \cap D_{\mathcal{M}_{t}}$ contains at least two distinct points on the normalization $\tilde{C}_{t}$ of $C_{t}$.

Proof. Since $X_{t}$ has Picard number 1, $C_{t}$ has non-empty intersection with $D_{\mathcal{M}_{t}}$. Suppose the intersection is only at one point on $\tilde{C}_{t}$. Then the normalization of $C_{t}-D_{\mathcal{M}_{t}}$ is biholomorphic to $\mathbf{C}$ and is simply connected. Since $F_{t}$ is unramified over $X_{t}-D_{\mathcal{M}_{t}}-Z_{t}$, this means that $\phi_{t}^{-1}\left(C_{t}\right)$ consists of $d_{t}$ distinct irreducible components, where $d_{t}$ is the degree of $\phi_{t}: F_{t} \rightarrow X_{t}$. This is a contradiction to Proposition 12 and the next lemma applied to each irreducible component $W$ of $F_{t}$.

Lemma 2. Let $\eta: W \rightarrow X$ be a generically finite morphism from an irreducible normal variety $W$ onto a Fano manifold $X$ with Picard number 1 which has a web $\mathcal{M}$. Suppose for a generic member $C$ of $\mathcal{M}$, each component of the inverse image $\eta^{-1}(C)$ is birational to $C$ by $\eta$. Then $\eta: W \rightarrow X$ itself is birational.

Proof. We may assume that $W$ is a projective manifold by desingularization. Suppose $\eta$ is not birational. Let $\mathcal{B} \subset X$ be the branch divisor of $\eta$ and $\mathcal{R} \subset W$ be the ramification divisor of $\eta$. By the assumption on $\eta$, each component $C_{1}$ of $\eta^{-1}(C)$ is a rational curve. By Proposition 6, $C_{1}$ has trivial normal bundle. So we have $K_{W} \cdot C_{1}=-2=K_{X} \cdot C=\eta^{*} K_{X} \cdot C_{1}$. This implies $C_{1}$ is disjoint from the ramification divisor $\mathcal{R} \subset W$. Since this holds for any component $C_{1}$ of $\eta^{-1}(C), C$ is disjoint from $\mathcal{B}$, a contradiction to the assumption that $X$ is of Picard number 1 .

Proposition 15. Fix a complex number $s, 0<|s|<\epsilon$. Under the assumptions of Proposition 14, for a generic choice of $C \subset X_{0}$ and an irreducible component $C^{\prime}$ of $f_{0}^{-1}(C)$, let $y_{1}, y_{2} \in C^{\prime}$ be any two points satisfying $f_{s}\left(y_{1}\right)=f_{s}\left(y_{2}\right)$. Then $f_{t}\left(y_{1}\right)=f_{t}\left(y_{2}\right)$ whenever $|t|<\epsilon$.

Proof. By continuity, it suffices to prove $f_{t}\left(y_{1}\right)=f_{t}\left(y_{2}\right)$ for $t$ satisfying $|t-s|<\delta$ for some positive real number $\delta<\min (|s|, \epsilon-|s|)$. By our choice of $C, C_{t}=f_{t}\left(C^{\prime}\right)$ is a member of $\mathcal{M}_{t}$ with trivial normal bundle in $X_{t}$. Since $X_{t}$ is Fano, $C_{t}$ is a rational curve. From Proposition 1 and Proposition 13 , we can choose a generic $C$ and $\epsilon>0$, so that $C_{t}=f_{t}\left(C^{\prime}\right)$ intersects $D_{\mathcal{M}_{t}}$ transversally for all $0<|t|<\epsilon$. By Proposition 14, there exists a simultaneous normalization $\mu_{t}: \mathbf{P}_{1} \rightarrow C_{t}$ for all $t$ satisfying $0<|t-s|<\delta$ so that $\mu_{t}(o)$ and $\mu_{t}(\infty)$ lie in $C_{t} \cap D_{\mathcal{M}_{t}}$ for two fixed distinct points $\{o, \infty\}$ in $\mathbf{P}_{1}$. Also, we can assume that $C^{\prime}$ is transversal to $E_{\mathcal{M}^{\prime}}$.

Let $\tilde{f}_{t}: \tilde{C}^{\prime} \rightarrow \mathbf{P}_{1}$ be the pull-back of $\left.f_{t}\right|_{C^{\prime}}$ to the normalizations. $\left\{\tilde{f}_{t},|t-s|<\delta\right\}$ gives a holomorphic family of meromorphic functions on $\tilde{C}^{\prime}$ with zeroes and poles lying over the points of $C^{\prime}$ intersecting $f_{t}^{-1}\left(\mu_{t}(o)\right)$ and $f_{t}^{-1}\left(\mu_{t}(\infty)\right)$. Since $f_{t}^{-1}\left(D_{\mathcal{M}_{t}}\right) \subset E_{\mathcal{M}^{\prime}}$ from Proposition 7 , these points 
are contained in the finite set $C^{\prime} \cap E_{\mathcal{M}^{\prime}}$ which does not depend on $t$. So this family of meromorphic functions must have the same zeroes and poles on $\tilde{C}^{\prime}$. Thus $\tilde{f}_{t}=h(t) \tilde{f}_{0}$ for some non-vanishing holomorphic function $h(t)$ on $\{|t-s|<\delta\}$, from which the assertion is obvious.

We are ready to prove:

Theorem 1. Let $\chi: \mathcal{X} \rightarrow \Delta:=\{t \in \mathbf{C},|t|<1\}$ be a regular family of Fano manifolds of Picard number 1 so that $X_{0}=\chi^{-1}(0)$ has rational curves with trivial normal bundles. For a given projective manifold $X^{\prime}$, suppose there exists a surjective morphism $f: \mathcal{X}^{\prime}=X^{\prime} \times \Delta \rightarrow \mathcal{X}$ respecting the projections to $\Delta$ so that $f_{t}: X^{\prime} \rightarrow X_{t}$ is a generically finite morphism for each $t \in \Delta$. Then there exists $\epsilon>0$ and a holomorphic family of biholomorphic morphisms $v_{t}: X_{o} \rightarrow X_{t},|t|<\epsilon$, satisfying $f_{t}=v_{t} \circ f_{o}, v_{0}=i d$.

Proof. By assumption, $X_{0}$ has a web $\mathcal{M}_{0}$. We will use the notation from Propositions 13-15.

Suppose that $f$ is birational. Then $f$ is biholomorphic over $\mathcal{X}-\mathcal{Z}$ where $\mathcal{Z}$ is a subvariety of codimension $\geq 2$. On $\mathcal{X}^{\prime}$ we have the vector field $\mathcal{V}$ lifting $\frac{d}{d t}$ on $\Delta . f_{*} \mathcal{V}$ is a vector field on $\mathcal{X}-\mathcal{Z}$. By Hartogs, we can extend it to a vector field on $\mathcal{X}$ which generates the required family of biholomorphic morphisms.

Suppose that $f_{t}$ is not birational, but generically $e$-to- 1 . We will construct a new projective manifold $\hat{X}$, a generically finite dominant rational map $\nu$ : $X^{\prime} \rightarrow \hat{X}$ and a holomorphic family of generically finite morphisms $g_{t}: \hat{X} \rightarrow$ $X_{t}$, so that $\nu$ is not birational and $f_{t}=g_{t} \circ \nu$ over generic points of $X^{\prime}$. This proves Theorem 1 by induction on $e$.

Fix $s$ as in Proposition 15. We say that a reduced 0 -cycle $y_{1}+\cdots+y_{k}$ of length $k$ on $X^{\prime}$ is special if $f_{s}\left(y_{1}\right)=\cdots=f_{s}\left(y_{k}\right)$ and we can find a sequence of points $z_{1}, \ldots, z_{m}$ so that $\left\{z_{1}, \ldots, z_{m}\right\}=\left\{y_{1}, \ldots, y_{k}\right\}$ as subsets of $X^{\prime}$ and there exist irreducible curves $l_{1}, \ldots, l_{m-1}$ which are members of the inverse image web of $\mathcal{M}_{0}$ so that $z_{i}, z_{i+1} \in l_{i}$ for $1 \leq i \leq m-1$. All special cycles have length $\leq e$ and there are special cycles of length $>1$ containing a generic point of $X^{\prime}$, otherwise $f_{t}$ is birational by Lemma 2 .

The set of all special cycles on $X^{\prime}$ gives a constructible subset of the Hilbert scheme of 0 -dimensional subschemes of $X^{\prime}$. We can find an irreducible component of this set so that the corresponding cycles cover an open set of $X^{\prime}$. Make such a choice with maximal possible length. Let $S$ be the closure of that component and let $\sigma: A \rightarrow S$ and $\lambda: A \rightarrow X^{\prime}$ be the universal family morphisms so that $\sigma$ is flat, but not birational. We claim that $\lambda$ is birational. 
Otherwise we have two distinct special cycles containing a given generic point $y$ of $X^{\prime}$, from which we can construct a special cycle of strictly longer length containing $y$, a contradiction to the choice of $S$.

Let $\hat{X}$ be a desingularization of $S$ and $\hat{\sigma}: \hat{A} \rightarrow \hat{X}, \hat{\lambda}: \hat{A} \rightarrow X^{\prime}$ be the induced morphisms. Define the rational map $\nu: X^{\prime} \rightarrow \hat{X}$ as $\nu:=\hat{\sigma} \circ \hat{\lambda}^{-1}$. Then $\nu$ is a generically finite dominant rational map which is not birational. Consider the morphism $\hat{f}_{t}=f_{t} \circ \hat{\lambda}$ from $\hat{A}$ to $X_{t}$. From Proposition 15, a generic fiber of $\hat{\sigma}$ is contained in a fiber of $\hat{f}_{t}$. Thus each fiber of $\hat{\sigma}$ is contained in a fiber of $\hat{f}_{t}$ by the flatness of $\hat{\sigma}$. It follows that we get a morphism $g_{t}: \hat{X} \rightarrow X_{t}$ satisfying $f_{t}=g_{t} \circ \nu$. Since $X^{\prime}, X_{t}$ are all projective, it is easy to see that $\left\{g_{t}\right\}$ is a holomorphic family.

An immediate consequence of Theorem 1 is:

Corollary 1. For any irreducible complete variety $X^{\prime}$ of dimension $\geq 3$, there are only countably many Fano manifolds of Picard number 1 having rational curves with trivial normal bundles, which can be the image of a generically finite morphism from $X^{\prime}$.

In [HM1], we showed that for any Fano manifold $S$ of Picard number 1 which is homogeneous, a finite morphism $f: S \rightarrow X$ onto a projective manifold $X$ must be a biholomorphism unless $X \cong \mathbf{P}_{n}$. It is natural to ask what happens if $S$ is almost homogeneous. We will consider a special case here. A Mukai-Umemura threefold (cf. [MU]) is a Fano threefold of Picard number 1 which is almost homogeneous under $P G L_{2}$. There are two such Fano threefolds, one having the octahedral group as the isotropy group, and the other having the icosahedral group as the isotropy group. Note that both the octahedral group and the icosahedral group are maximal finite subgroups in $P G L_{2}$ (e.g. $[\mathrm{YY}]$ ).

Corollary 2. Let $S$ be a Mukai-Umemura threefold and $X$ be a smooth variety of positive dimension. If $f: S \rightarrow X$ is a surjective morphism, then either $X \cong \mathbf{P}_{3}$ or $f$ is biholomorphic.

Proof. Since $S$ is of Picard number $1, f$ is a finite morphism and $X$ is a Fano threefold. If $X$ is different from $\mathbf{P}_{3}$, it is either the 3-dimensional hyperquadric or a Fano threefold having a rational curve with trivial normal bundle. The $P G L_{2}$-action on $S$ descends to $X$ by [HM2] for the hyperquadric and by Theorem 1 for the others. Thus $X$ is almost homogeneous under $P G L_{2}$, and the isotropy subgroup is a finite subgroup of $P G L_{2}$ containing the isotropy subgroup for $S$. However, the isotropy subgroup for $S$, either the octahedral group or the icosahedral group, is a maximal finite subgroup of $P G L_{2}$, a contradiction unless $f$ is 1-to- 1 . 


\section{Boundedness of degrees of finite morphisms}

In this section we will prove:

Theorem 2. Let $X$ and $X^{\prime}$ be $n$-dimensional Fano manifolds of Picard number 1. Assume that $X$ has rational curves with trivial normal bundles. Then there exists a positive number $N$ determined by $X^{\prime}$ such that for any finite morphism $f: X^{\prime} \rightarrow X$, the degree of $f$ is bounded by $N$.

In fact, $N$ can be calculated from the degrees of extended discriminantal divisors and discriminantal orders of webs on $X^{\prime}$, together with Chern numbers of $X^{\prime}$ and $X$. Note that there are only finitely many webs on $X^{\prime}$ and $X$ :

Proposition 16. On a Fano manifold, there are only finitely many webs.

Proof. Any curve with trivial normal bundle on a Fano manifold must be a rational curve and its degree with respect to the anti-canonical bundle is 2 . Since the anti-canonical bundle is ample, we get the finiteness of irreducible webs. Since a web is just the finite union of irreducible webs, there are only finitely many webs.

Proposition 17. Let $X^{\prime}$ be an n-dimensional Fano manifold. Then there exists a positive number $N_{1}$ determined by $X^{\prime}$ with the following property: for any finite morphism $f: X^{\prime} \rightarrow X$ onto a Fano manifold $X$ which has a web $\mathcal{M}$ and for any component $L^{\prime}$ of $f^{-1}\left(D_{\mathcal{M}}\right)$, the local sheeting number of $f$ at a generic point of $L^{\prime}$ is bounded by $N_{1}$.

Proof. The local sheeting number is bounded by a number determined by the discriminantal orders of $L^{\prime}$ from Proposition 9. Since there are only finitely many possibilities of $\mathcal{M}^{\prime}$ from Proposition 16 , there are only finitely many possibilities for $L^{\prime}$. Thus we can find a bound $N_{1}$.

Proof of Theorem 2. Choose a web $\mathcal{M}$ on $X$ and let $C$ be a generic member of $\mathcal{M}$. $C$ is an immersed rational curve. For a given finite morphism $f: X^{\prime} \rightarrow$ $X$, let $\mathcal{M}^{\prime}$ be the inverse image web of $\mathcal{M}$. Let $C^{\prime}$ be a member of $\mathcal{M}^{\prime}$ so that $f\left(C^{\prime}\right)=C$. Let $h: \tilde{C}^{\prime} \rightarrow \tilde{C}$ be the the pull-back of $\left.f\right|_{C}$ to the normalization.

We may assume that $C$ (resp. $C^{\prime}$ ) intersects $D_{\mathcal{M}}$ (resp. $E_{\mathcal{M}}$ ) transversally. For $z \in \tilde{C}^{\prime}$, let $e_{z}$ be the ramification index of $h$ at $z$. If $r_{z}$ is the local sheeting number of $f$ at $\mu(z)$ where $\mu: \tilde{C}^{\prime} \rightarrow X^{\prime}$ is the normalization onto its image, 
then $e_{z}=r_{z}-1$. For $x \in \tilde{C}$, let $\left|h^{-1}(x)\right|$ be the number of points of $\tilde{C}^{\prime}$ over $x$. When $m$ is the degree of $h$,

$$
\begin{aligned}
\sum_{z \in \tilde{C}^{\prime} \cap f^{-1}\left(D_{\mathcal{M}}\right)} e_{z} & =\sum_{x \in \tilde{C} \cap D_{\mathcal{M}}}\left(m-\left|h^{-1}(x)\right|\right) \\
& =m C \cdot D_{\mathcal{M}}-\sum_{x \in \tilde{C} \cap D_{\mathcal{M}}}\left|h^{-1}(x)\right| \\
& =m C \cdot D_{\mathcal{M}}-C^{\prime} \cdot f^{-1}\left(D_{\mathcal{M}}\right) \\
& \geq 2 m-C^{\prime} \cdot E_{\mathcal{M}^{\prime}}
\end{aligned}
$$

where we used $f^{-1}\left(D_{\mathcal{M}}\right) \subset E_{\mathcal{M}^{\prime}}$ from Proposition 7 and $C \cdot D_{\mathcal{M}} \geq 2$ from Proposition 14. The readers can easily see below that for the proof of Theorem 2 , the obvious inequality $C \cdot D_{\mathcal{M}} \geq 1$ is, in fact, sufficient. From Proposition 17 ,

$$
\begin{aligned}
\sum_{z \in \tilde{C}^{\prime} \cap f^{-1}\left(D_{\mathcal{M}}\right)} e_{z} \leq \sum_{z \in \tilde{C}^{\prime} \cap f^{-1}\left(D_{\mathcal{M}}\right)}\left(N_{1}-1\right) \\
\leq\left(N_{1}-1\right) C^{\prime} \cdot E_{\mathcal{M}^{\prime}}
\end{aligned}
$$

It follows that $m \leq \frac{N_{1}}{2} C^{\prime} \cdot E_{\mathcal{M}^{\prime}}$.

From Proposition 16, there are only finitely many webs on $X^{\prime}$. Consider the union $\mathcal{L}$ of extended discriminantal divisors of all the webs on $X^{\prime}$. Let $\mathcal{L}=N_{2} K_{X^{\prime}}^{-1}$ in $\operatorname{Pic}\left(X^{\prime}\right) \otimes \mathbf{Q}$ for some positive rational number $N_{2}$. Then $C^{\prime} \cdot E_{\mathcal{M}^{\prime}} \leq C^{\prime} \cdot \mathcal{L} \leq 2 N_{2}$. Thus $m \leq N_{1} N_{2}$.

Let $\hat{R} \subset X^{\prime}$ be the ramification divisor $R$ of $f$ with the multiplicity given by the ramification indices of each component. Then $\hat{R}=f^{*} K_{X}^{-1}-K_{X^{\prime}}^{-1}$ and $\hat{R} \cdot C^{\prime}=2 m-2$. It follows that $\hat{R}=(m-1) K_{X^{\prime}}^{-1}$ and $f^{*} K_{X}^{-1}=K_{X^{\prime}}^{-1}+\hat{R}=$ $m K_{X^{\prime}}^{-1}$ in $\operatorname{Pic}\left(X^{\prime}\right)$. Thus

$$
\operatorname{deg}(f)=\frac{\left(f^{*} K_{X}^{-1}\right)^{n}}{\left(K_{X}^{-1}\right)^{n}}=m^{n} \frac{\left(K_{X^{\prime}}^{-1}\right)^{n}}{\left(K_{X}^{-1}\right)^{n}} \leq\left(N_{1} N_{2}\right)^{n}\left(K_{X^{\prime}}^{-1}\right)^{n} .
$$

As an immediate consequence, we obtain:

Corollary 3. A non-constant self-morphism $f: X \rightarrow X$ of a Fano manifold of Picard number 1 which has rational curves with trivial normal bundles is an isomorphism.

Proof. If $f$ has degree $d>1$, the $m$-th successive composition $f^{m}: X \rightarrow X$ gives a finite morphism of degree $d m$, where $m$ can be arbitrary large; a contradiction to Theorem 2 .

Now we obtain the following partial answer to the Question discussed in the introduction of this paper.

Corollary 4. Let $X^{\prime}$ be a Fano manifold of Picard number 1. Amid Fano manifolds of Picard number 1 which have rational curves with trivial normal 
bundles, only finitely many can be the image of a holomorphic map from $X^{\prime}$. Furthermore, for each such $X$ there are at most a finite number of non-trivial holomorphic maps $f: X^{\prime} \rightarrow X$ up to automorphisms of $X$.

Proof. Suppose there exists an infinite sequence $f_{i}: X^{\prime} \rightarrow X_{i}$ of holomorphic maps to Fano manifolds $X_{i}$ of Picard number 1 which have rational curves with trivial normal bundles. By the boundedness of Fano manifolds of a given dimension $(\overline{\mathrm{Kl}})$, we may assume that $X_{i}$ 's are members of an irreducible flat family of Fano manifolds. By a pluri-anti-canonical embedding, we can regard $X_{i}$ 's as submanifolds in some projective space $\mathbf{P}_{N}$ with the same Hilbert polynomial. The graphs of $f_{i}$ 's are Fano submanifolds in $X^{\prime} \times \mathbf{P}_{N}$, which are of bounded degree with respect to $K_{X^{\prime}}^{-1} \otimes \mathcal{O}(1)$ from Theorem 2, where $\mathcal{O}(1)$ denotes the hyperplane bundle on $\mathbf{P}_{N}$. Thus there are finitely many irreducible families of subvarieties in $X^{\prime} \times \mathbf{P}_{N}$, so that the graphs of $f_{i}$ 's are dense in each family. Generic members of such a family must be isomorphic to $X^{\prime}$ under the projection $X^{\prime} \times \mathbf{P}_{N} \rightarrow X^{\prime}$. Thus they give a continuous family of finite morphisms from $X^{\prime}$ to a family of Fano manifolds having rational curves with trivial normal bundles and must be related by automorphisms of the images by Theorem 1 . It follows that there are only finitely many isomorphism classes of $f_{i}$ 's up to automorphisms of the images.

\section{References}

[Am] Amerik, E., Maps onto certain Fano threefolds. Documenta Mathematica 2 (1997) 195-211.

[ARV] Amerik, E., Rovinsky, M. and Van de Ven, A., A boundedness theorem for morphisms between threefolds, Annal. L'Institut Fourier 49 (1999) 405-415.

[HM1] Hwang, J.-M. and Mok, N., Holomorphic maps from rational homogeneous spaces of Picard number 1 onto projective manifolds. Invent. math. 136 (1999) 209-231.

[HM2] Hwang, J.-M. and Mok, N., Varieties of minimal rational tangents on uniruled manifolds. in Several Complex Variables, ed. by M. Schneider and Y.-T. Siu, MSRI Publications 37, Cambridge University Press (2000) 351-389.

[HM3] Hwang, J.-M. and Mok, N., Cartan-Fubini type extension of holomorphic maps for Fano manifolds of Picard number 1, J. Math. Pures Appl. 80 (2001) 563-575.

[IS] Iliev, A. and Schuhmann, C., Tangent scrolls in prime Fano threefolds. Kodai Math. J. 23 (2000) 411-431.

[Is] Iskovskikh, V.A., Anticanonical models of 3-dimensional algebraic varieties. J. Soviet Math. 13 (1980) 745-814.

[Kd] Kodaira, K., On stability of compact submanifolds of complex manifolds. Amer. J. Math. 85 (1963) 79-94.

[Kl] Kollár, J., Rational curves on algebraic varieties. Ergebnisse der Mathematik und ihrer Grenzgebiete, 3 Folge, Band 32, Springer Verlag, 1996.

[KO] Kobayashi, S. and Ochiai, T., Meromorphic mappings onto compact complex spaces of general type. Invent. math. 31 (1975) 7-16. 
[Ma] Maehara, K., A finiteness property of varieties of general type. Math. Ann. 262 (1983) 101-123.

[MU] Mukai, S. and Umemura, H., Minimal rational threefolds. in Algebraic Geometry, Tokyo/Kyoto 1982. Lecture notes in Math. 1016 (1983) 490-518.

[Sc] Schuhmann, C., Morphisms between Fano threefolds J. Alg. Geom. 8 (1999) 221-244

[YY] Yau, Stephen S.-T. and Yu, Y., Gorenstein quotient singularities in dimension three, Memoirs AMS 105 (1993).

Korea Institute for Advanced Study, 207-43 Cheongryangri-dong, Seoul 130012, Korea

E-mail address: jmhwang@ns.kias.re.

Department of Mathematics, The University of Hong Kong, Pokfulam Road, HONG KONG

E-mail address: nmok@hkucc.hku.hk 